(REVIEW ARTICLE)

\title{
Transmigration of mandibular canines - A review of the literature and a report of five cases
}

\author{
Kumar Santosh, Bajjad A Adeel *, Raj Yukti, Tiwari K Dhruv and Agarwal Malvika
}

Department of Orthodontics and Dentofacial Orthopedics, Kothiwal Dental College and Research Centre, Moradabad, UP244001, India.

Publication history: Received on 05 June 2020; revised on 12 June 2020; accepted on 14 June 2020

Article DOI: https://doi.org/10.30574/wjarr.2020.6.3.0194

\begin{abstract}
Transmigration of the mandibular canine is very uncommon, the etiology of which isn't clear. A cuspid is classified as "transmigrated" when it cross the mid-line. We present five instances of transmigrated mandibular canines together with an audit of the writing etiology, clinical highlights, treatment alternatives, case reports and conclusion at the end.
\end{abstract}

Keywords: Canine; Mandibular; Ectopic; Transmigration

\section{Introduction}

Impacted teeth are critical to dentistry and are especially noteworthy in orthodontics, particularly if the affected tooth is a canine. In the human dentition, maxillary and mandibular canines are solid teeth and are extremely significant from a stylish just as a practical perspective when they are available in their ordinary position. Shah et al. discovered eight unerupted mandibular canines in 7886 individuals [1]. Grover and Lorton found impacted mandibular canines in 5000 people [2]. An unerupted tooth at times moves to an area some good ways from the site wherein it developed, however it as a rule stays inside a similar side of the curve. The mandibular changeless canine is the main tooth in the dental arch answered to move over the midline. Javid discovered one such case in 1000 understudies [3]. In doing as such, the tooth as a rule goes along the labial side of the incisor roots and relocates similar to the underlying foundations of the main molar on the contrary side. Ando et al. were the first to utilize the term "transmigration" [4]. While assessments of the recurrence of affected maxillary canines are somewhere in the range of 2.06 and 3.53\%, appraisals of the recurrence of mandibular transmigration extend from 0.075 to $0.34 \%$.

\section{Etiology}

As indicated by Cakan et al. transmigration is a dental phenomenon of "partly environmental and partly genetic"etiology [5]. The polygenic nature of dental manifestations makes linkage to just one deformity unthinkable. At the point when varieties are seen among the individuals from a family, they regularly express various powers of related deviations. As innovation keeps on improving, the proposal of hereditary meeting turns out to be increasingly conceivable, truth be told, progressively prompted.

Several other factors that that are believed to be involved in transmigration, such as premature loss of deciduous teeth, retention of the deciduous canine, odontomas, crowding, supernumerary teeth, and excessive crown length of the mandibular canine. Be that as it may, the etiology and definite mechanism are as yet indistinct. The mandibular left canine is influenced more regularly than the right and migrates more in females than in males (1.6:1 ratio) [6]. Unilateral migration is more typical than bilateral migration. Bilateral migration of mandibular canines may occur regardless of having sufficient space for eruption. Transmigrating teeth can cause root resorption, tipping, and sensitivity of adjacent teeth and pain or distress for the patient. The side effects are identified to the severity of the transmigration.

\footnotetext{
${ }^{*}$ Corresponding author: Bajjad A Adeel
} 
According to Mupparapu's classification, there are five types of transmigrated mandibular canine [Figure 1], with the type 1 being most frequent [7]. In this manner, early determination and evaluation of the degree of canine transmigration are urgent for the counteraction of impaction, and they could diminish related complexities in both esthetics and function. Conversely, failure to diagnose, manage, or appropriately treat impacted canines can lead to external resorption of adjacent teeth [especially the lateral incisors], esthetic problems, misalignment of neighboring teeth, shortened dental arches, and increased follicular cyst formation and recurrent infections bringing about irreversible harm that may eventually cause tooth loss.

Ando et al. found the premature loss of teeth, inadequate space, and excessively large crowns are the etiological factors [4]. In any case, premature extraction of the deciduous canine is practiced in an attempt to correct the eruption of an ectopic permanent canine.

Costello et al. and Joshi noticed several cases where the deciduous canine had been retained [8,9]. A few cases of transmigrated canines occurred related to hypodontia and overabundance space.

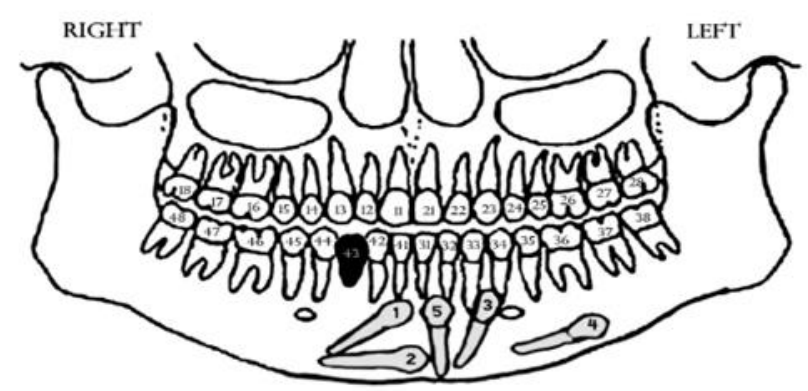

Figure 1 Mupparapu's classification

In 2002, Mupparapu classified the pattern of intraosseous transmigration of impacted mandibular canine as follows:

- Type-1 - Canine impacted mesioangularly across the midline, labial or lingual to the anterior teeth

- Type-2 - Canine impacted horizontally near the lower border of the mandible, inferior to the apices of the incisors

- Type-3 - Canine erupted either mesial or distal to the contralateral canine

- Type-4 - Canine impacted horizontally near the inferior border of the mandible, below the apices of either premolars or molars on the opposite side

- Type-5 - Canine positioned vertically in the midline, with the long axis of the tooth.

The role of crowding and spacing in the etiology of ectopic canines is hard to decide. . Be that as it may, transmigration has been accounted for to happen in the two circumstances. Joshi contradict with the idea of lower incisor proclination and enlargement of the symphyses as etiologic factors. He believed that this is a result of canine migration, not a reason [9]. Kerr suggested that the increase in lower incisor proclination might be because of normal variations of incisor angulation during development [10].

Alaejeos-Algarra et al. expressed that canine tooth germs are found further from the normal site of eruption than are germs of other teeth [11]. In spite of the fact that this is valid for maxillary canines, it is not so in the mandible. An anomalous position of the tooth germ may likewise be engaged with the pathogenesis of canine transmigration. Be that as it may, all accessible proof focuses to the tooth bud developing in its normal place and subsequently migrating to an ectopic position. Mitchell, Nixon and Lowley submitted reports of cases in which the plausible etiology of the displacement of a lower canine was a mandibular fracture through the developing crypt [12,13]. Despite the fact that the relationship of of the trauma and the displacement of the lower left canine could be coincidental, this appears to be impossible. This is especially obvious given the corresponding developmental positions of the two mandibular canines at the time of the injury and the site of the fracture line through the crypt of the left mandibular canine. However, Ranta and Ylipaavalniemi, in a study on the effect of jaw fractures in children on the consequent development of permanent teeth, found that teeth in which root formation had just begun at the time of fracture appeared to erupt normally yet showed shorter roots than unaffected contra lateral teeth [14]. This was apparently because of severance or impairment of the vascular supply to the pulp. None of the teeth in their study showed a deviant eruption path. 
Shapira et al. in their studies showed three cases of transmigration, one of which was accompanied by an odontoma and they attributed the displacement of the canine directly to the presence of the odontoma [15]. Shapira and Kuftinec reviewed cases of transmigrated canines and revealed that seven cases (9.5\%) showed hypodontia, whereas five cases (7\%) with odontomas were found. In 3 of these (4\%), both anomalies were present [16].

Taguchi et al. introduced a series of 15 cases, six of which showed hyperdontia [17]. No notice of related hypodontia is made; however, one figure oviously shows a missing lower lateral incisor with a fused predecessor. They mentioned odontomata as a reference of disturbance of eruption, reporting considerable improvement in the position of those canines associated with an odontoma, after removal of the same. In any case, the surgical procedure itself could have had a beneficial effect, similar to the effect of extraction of primary canines or crown exposure on the eruption path of ectopic maxillary canines.

\section{Clinical features}

- Retained primary tooth.

- Deviated midline.

- Absent permanent tooth.

- Lateral incisor agenesis.

- Incisor proclination.

- $\quad$ Resorption of adjacent tooth (root).

- Tipping \& sensitivity of adjacent teeth.

- Pain or distress for the patient (rarely).

\section{Management of transmigrated mandibular canines}

\subsection{Treatment consideration}

- Location

- Angulation

- Condition

\subsection{Treatment options-}

- $\quad$ Preventive \& Interceptive Treatment.

- $\quad$ Radiographic monitoring

- Surgical removal

- Transplantation.

- Exposure and orthodontic alignment.

- Observation.

\subsubsection{Preventive and Interceptive Treatment}

An odontoma or a small root fragment or interferes with the normal path of the eruption of the tooth, henceforth their removal would facilitate its eruption. Taguchi et al. revealed, after removal of the odontoma and surgical exposure an improvement in the position of the associated canines [17]. Vichi and Franchis recommended that an 8-to 9-year-old patient with an excessive mesial inclination of the unerupted mandibular permanent canine should be kept under basic perception with periodical panoramic radiographic examination [18]. If the position of the unerupted mandibular canine is seen to progressively tilt more to the mesial, interceptive measures ought to be taken. The preventive and interceptive treatment incorporates extraction of the retained deciduous canine and surgical exposure of impacted canine followed by orthodontic treatment. This stresses the importance of early diagnosis to correct this problem before the tooth migrates excessively far from its original location. 


\subsubsection{Radiographic Monitoring}

Transmigrated tooth can be left in place if it is symptomless and not related with any pathology. A series of periodic radiographs sought to be taken to check the status of the transmigrated tooth. A persistent exacerbating of position or development of cystic lesion and in case of severe root resorption of adjacent teeth, surgical removal is indicated.

\subsubsection{Surgical removal}

Careful extraction has all the earmarks of being the most preferred treatment for moved canines, as opposed to a brave exertion to take the tooth back to its original spot. This is particularly obvious when the mandibular arch is crowded and requires therapeutic extractions to correct the incisor crowding. Thoma expressed that transmigrated canines usually have to be removed [19]. Fiedler and Alling also recommend the extraction of transmigrated canines [20].

\subsubsection{Transplantation}

On the off chance that the transmigrated canine is in great situation for careful evacuation in one piece asymptomatically and there is adequate space with retained deciduous canine transplantation may be undertaken. Timing of transplant is significant since the primary objective is to obtain maximum root length. The best time to perform the procedure is when the root length of the tooth is between one-half and three fourths complete for the restoration of blood supply. The prognosis is diminished as the root apex approaches closure. The time span from removal to reinsertion ought to be minimal; ideally, this is a nonstop relocation and desiccation of the periodontal ligament can cause resorption, ankylosis, and failure. Retrograde endodontic treatments are performed simultaneous to transplantation with differing degrees of success; such treatment brings a foreign substance into the site, possibly initiating inflammatory reaction, but it greatly increases the length of the procedure and time the transplant stays out of the mouth. Such control will assuredly traumatize the root surface. It would progressively reasonable to perform the root canal treatment after the periodontal ligament attachment has readapted if such treatment demonstrates essential.

\subsubsection{Exposure and orthodontic alignment.}

Wertz used orthodontic treatment to bring labially impacted transmigrated canine into its correct position [21].

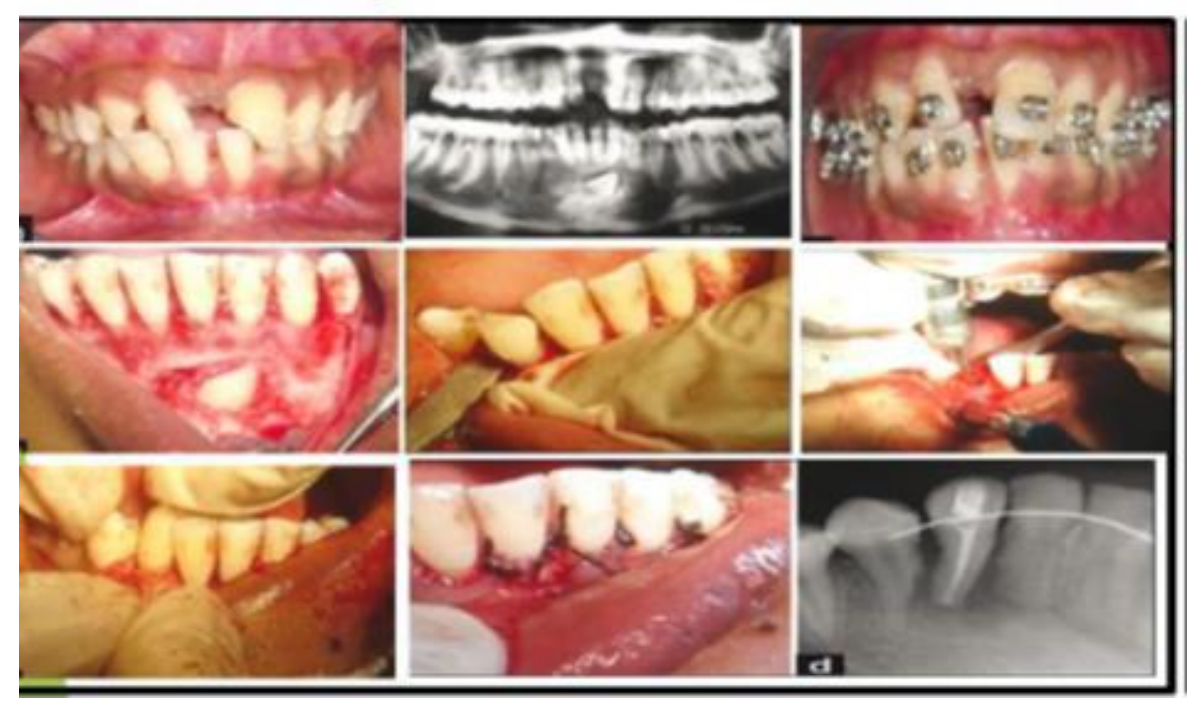

Figure 2 Autotransplantation of 43.

However, if the crown of such a tooth migrates past the opposite incisor area or if the apex apparently to have migrated past the apex of the adjacent lateral incisor, it might be precisely difficult mechanically to bring it into place by mechanically. Abbott et al. depicted the transposition of an incompletely erupted permanent right canine to a position between the permanent left canine and the left lateral incisor and showed that the tooth was amenable to orthodontic treatment [22]. They recommended that the premature extraction of first premolars ought to be avoided when radiographs shows the presence of an overly mesially angulated unerupted canine that has begun to migrate labially across the incisors. In these cases, it might be difficult to bring the canine to its correct position. Taguchi et al. detailed considerable improvement in the position of those canines associated with an odontoma, after careful removal of the odontoma and surgical exposure [17]. 


\subsubsection{Observation}

It has been upheld that an unerupted impacted tooth be removed when helpful. Different authors believed that symptomless, non-erupted teeth can be left in place. In these patients, a series of successive radiographs ought to be taken periodically. A dynamic exacerbating of the position of the unerupted canine or suggestion of cystic difference in the follicle should lead the clinician to consider the possibility of surgical extraction. The presence of pressure resorption of the roots of adjacent teeth, periodontal disturbances, or other possible foci for the spread of infection, prosthetic problems, malposition of the adjacent teeth, and neuralgic symptoms have been incorporated as indications for surgical intervention in instances of impacted mandibular canines.

\section{Case reports}

Following five cases have been reported in the Department of Orthodontics \& Dentofacial Orthopaedics, Kothiwal Dental College \& Research Centre, Moradabad since last 3 year.

\subsection{Case 1}

A 20 year male patient with class I molar relationship bilaterally. Clinically, a retained 73 was present and radiographic examination revealed a Type 1 transmigrated mandibular left permanent canine. Treatment planning involved extraction of deciduous canine and orthodontic traction of 33 followed by levelling and alignment [Figure 3].
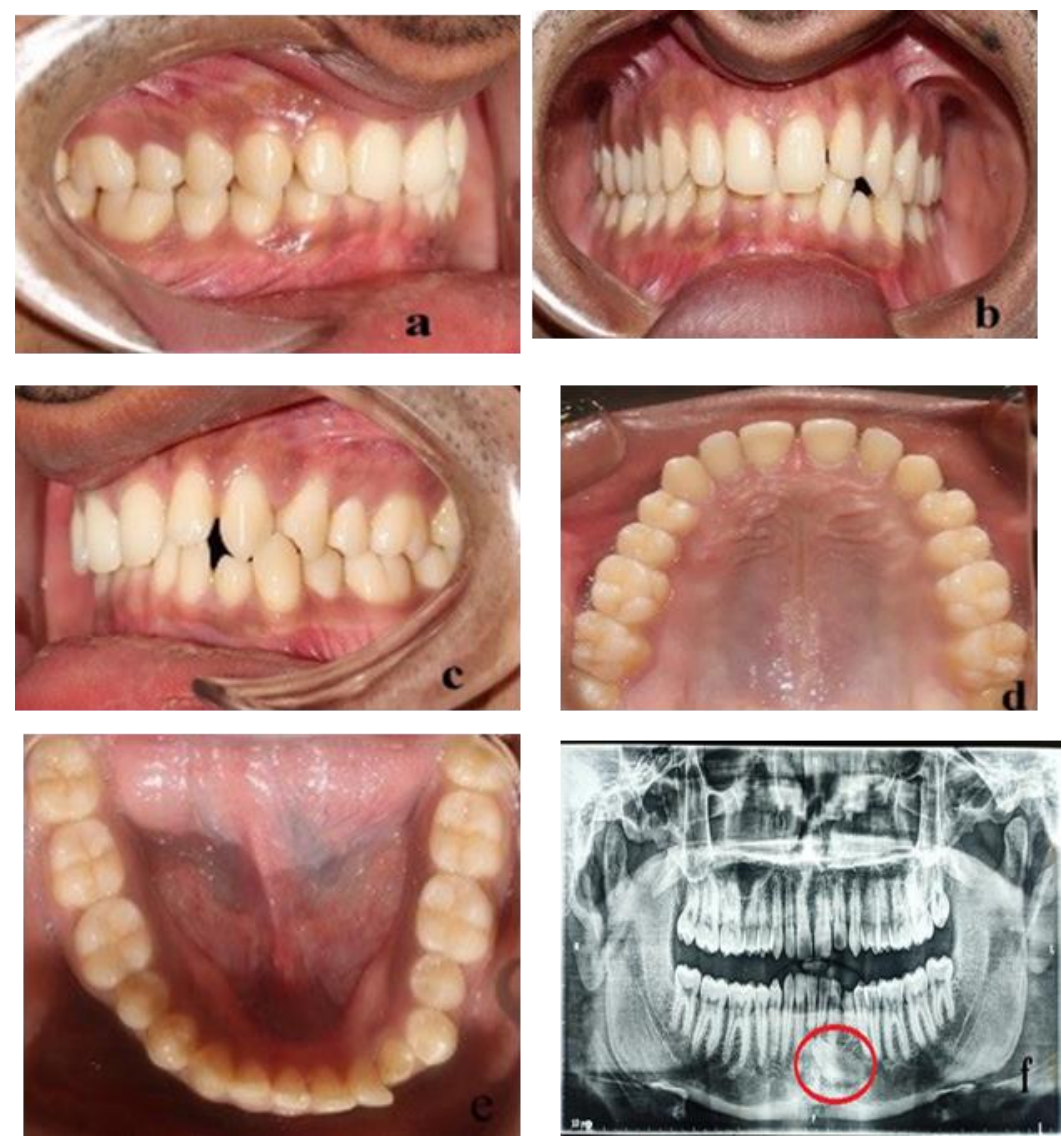

Figure 3 Transmigrated 33

\subsection{Case 2}

A 17 year old male patient with a chief complaint of lingually placed teeth in mandibular anterior toot region. On Clinical examination, a lingually placed 32 was present that was removed surgically. On performing radiographic examination, a Type 4 transmigrated mandibular right canine (43) was found in the lower border of the mandible. Treatment planning was decided to surgically extract 43 because of its poor prognosis followed by levelling and alignment [Figure 4]. 

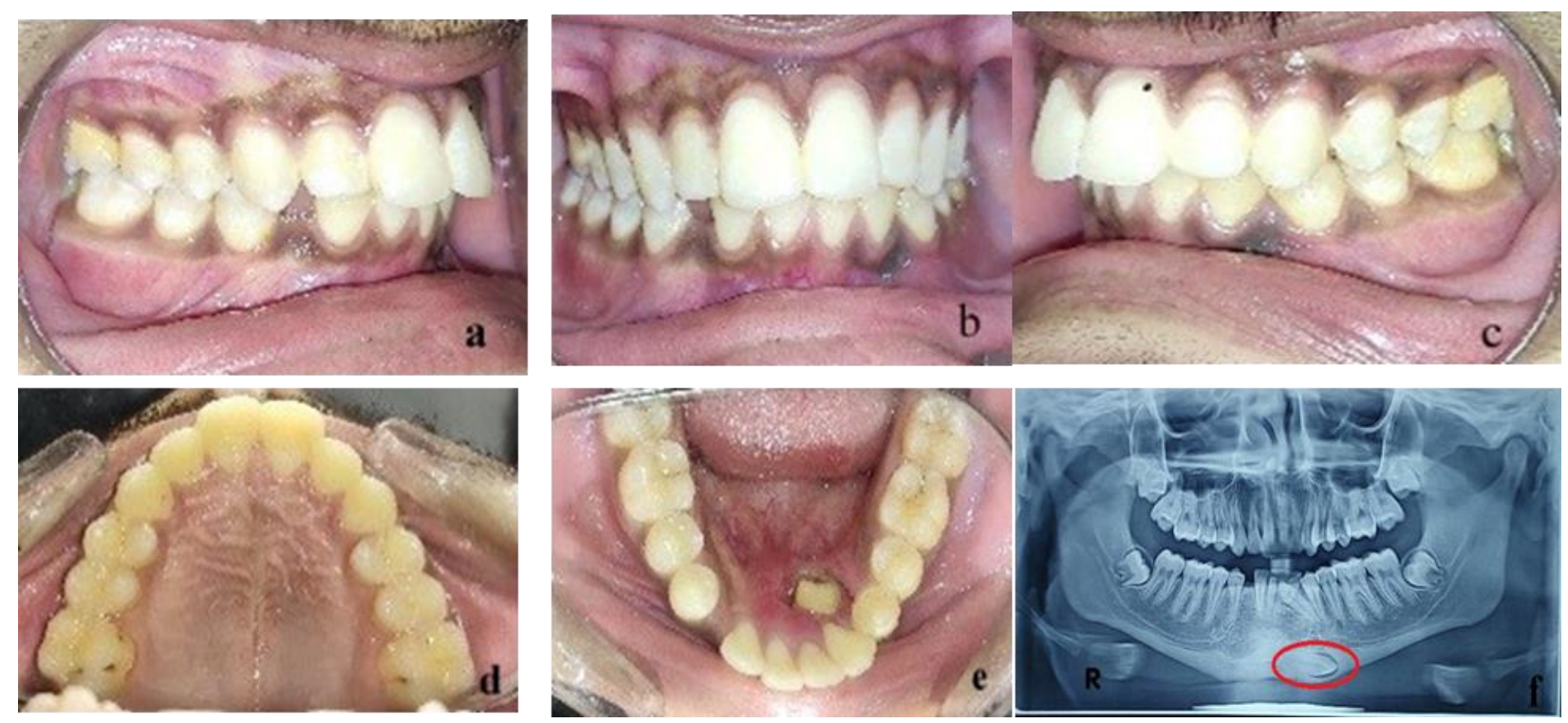

Figure 4 Transmigrated 43

Case 3 [Figure 5]:A 15 year old patient with proclined and protruded upper and lower incisors having end on molar relationship bilaterally with retained 73 and 83. A bulge was seen in the mandibular anterior vestibular region. Radiographically Type 2 transmigrated mandibular right canine was seen which cross the midline. So the treatment planning involved eruption of 43 was along with extraction of 14,24,35,45,73 and 83 for achieving a class I molar relationship bilaterally.
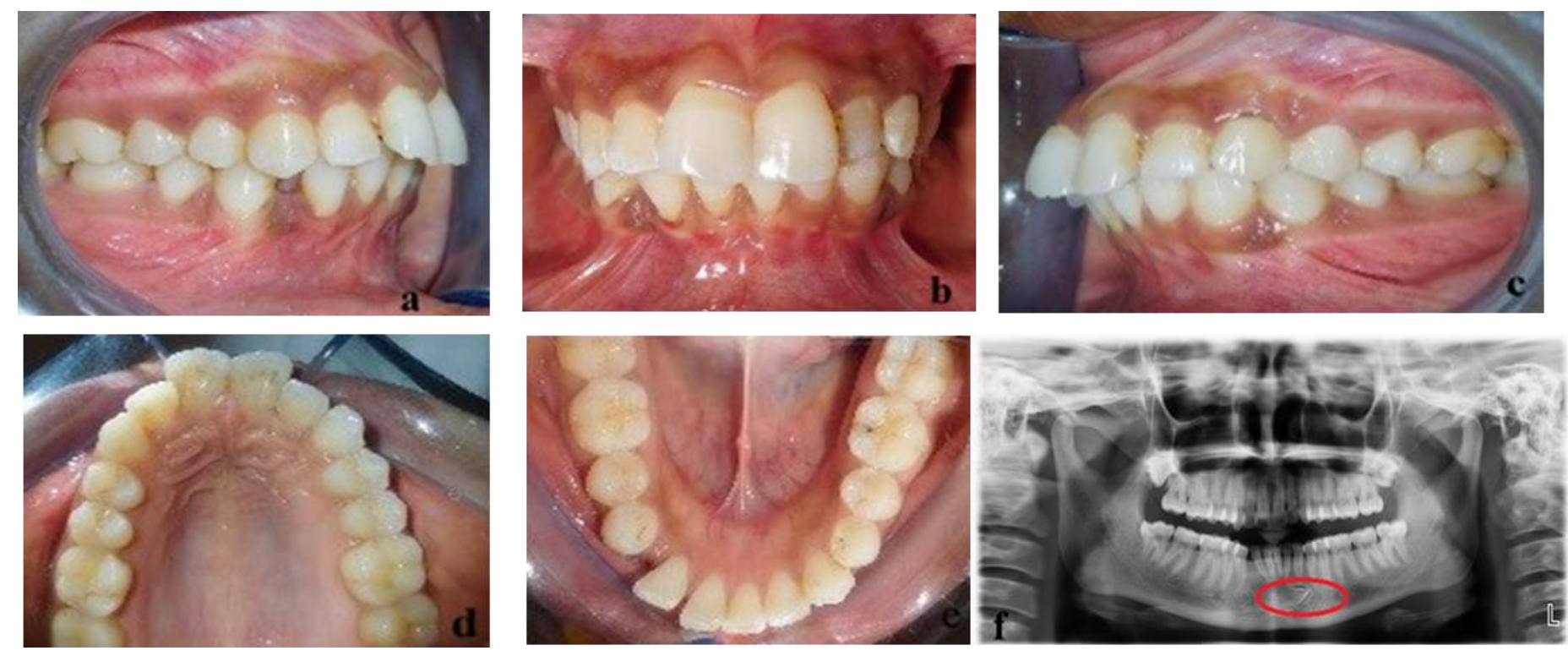

Figure 5 Transmigrated 43

\subsection{Case 4}

A 22-year-old patient with Bimaxillary protrusion came to the department with chief complaint of proclined upper and lower front teeth. On examination, her lower right canine was found to be missing. Radiographic examination revealed that it was transmigrated to the opposite side of the arch. As the transmigrated canine was Type 3, so her treatment planning included extraction of upper 1st premolars, lower left 1st premolar and lower right transmigrated impacted canine. The case was finished maintaining the class I molar relation bilaterally with proximal stripping of lower right premolars $(1 \mathrm{~mm})$ for achieving good buccal intercuspation and concedent midline [Figure 6]. 

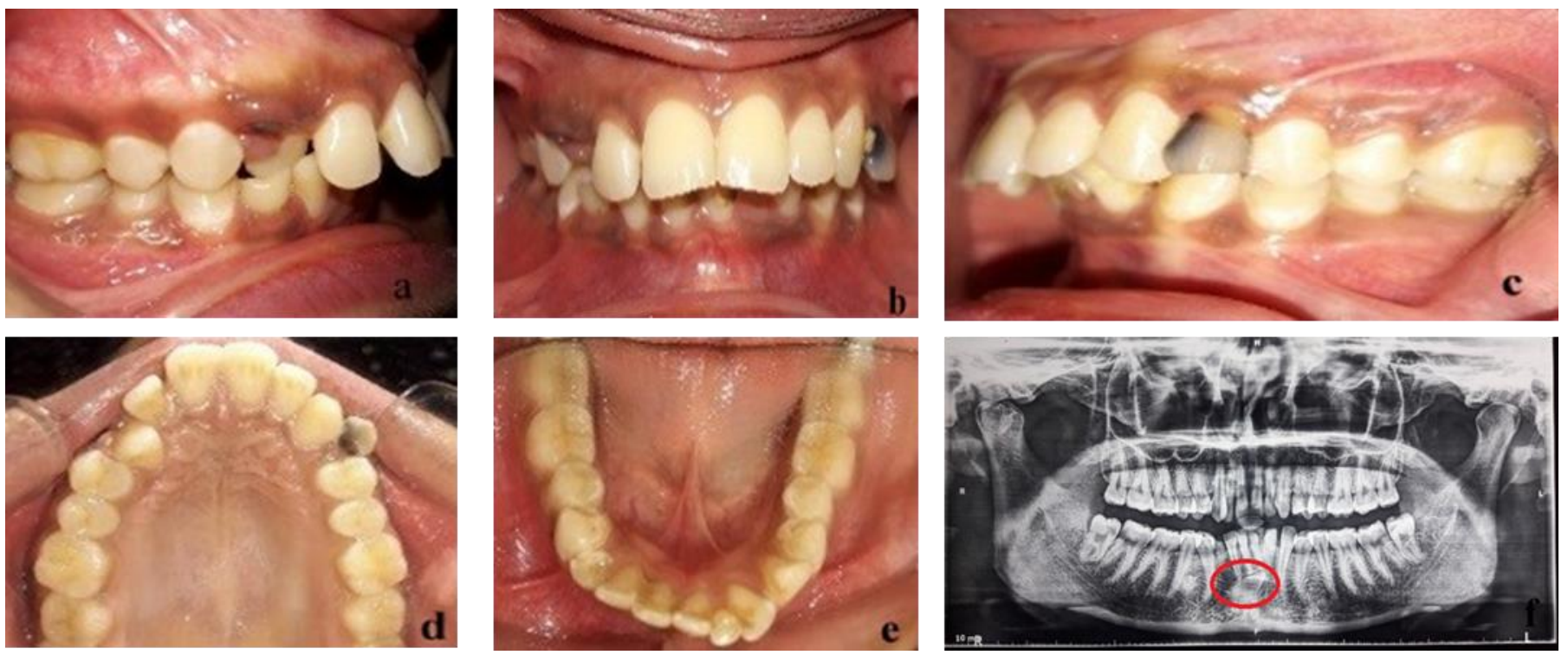

Figure 6 Transmigrated 43.

\subsection{Case 5}

A 22 year old patient with proclined and protruded anterior teeth having class I molar relation bilaterally. A retained 73 and missing 33 was seen clinically. So after performing radiographic examination a Type 4 transmigrated canine was found in the lower border of the mandible which was horizontally placed. Transplantation of 33 was planned but patient refused this treatment plan because of surgical procedure involved and it might have a questionable prognosis so finally 14,24, 73 and 44 was extracted followed by levelling and alignment [Figure 7].

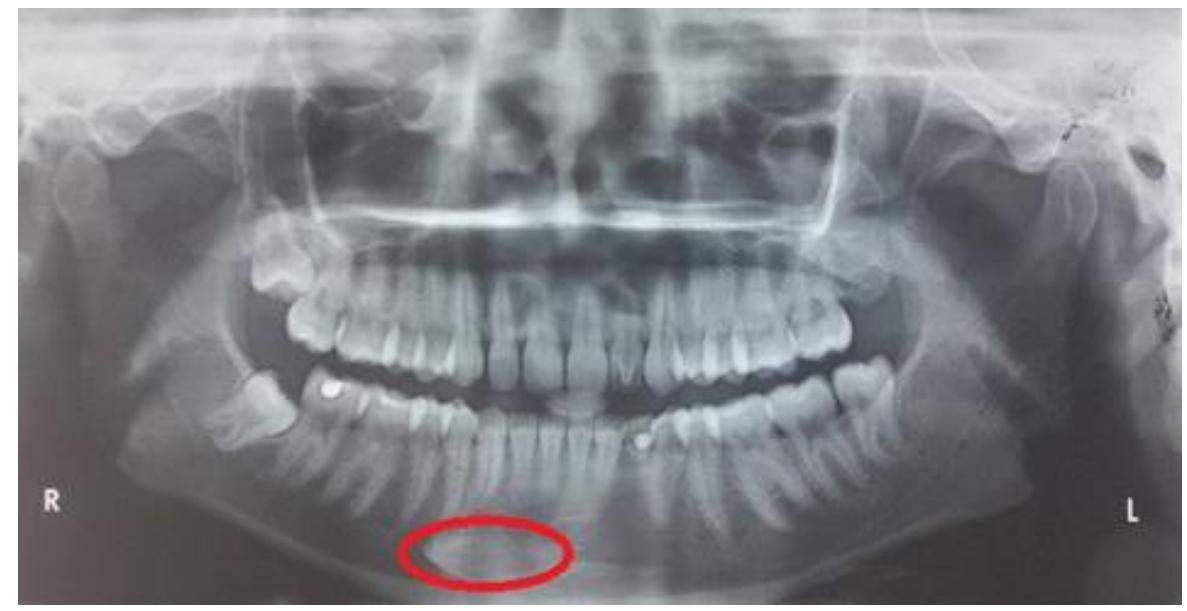

Figure 7Transmigrated 33.

\section{Conclusion}

Transmigration of the mandibular canine across the mandibular midline is an exceptional marvel described in the dental literature. More often than not, the canine just migrates without any pathological entity yet in a couple of casesa cyst or odontoma accompanies such a tooth. More often than not there are no symptoms, and such teeth have been found at the time of radiological examination before orthodontic treatment. Since the advent of panoramic radiography, such migratory teeth have been found more frequently. Etiology of this marvel is dark. In spite of this heredity, trauma to the mandible at a very early age of the patient, and a very small obstacle, such as root fragment, could be adequately to divert such a tooth to an abnormal path.

Extraction of the transmigrated canine seems, by all accounts, to be the main decision of treatment in the majority of the cases. Regardless, in an exceptional conditions when other teeth are in normal position and the space for the 
relocated canine is adequate, a transplantation methodology is one method for treating such cases. It is important to take care to anesthetize the transmigrated tooth from the side where it began. Be that as it may, if the careful extraction is done under general sedation, this issue doesn't emerge.The nearness of an over held mandibular deciduous canine ought to consistently be explored radiographically. An intraoral radiograph is normally not adequate, and it ought to constantly be enhanced with an occlusal and extraoral radiograph, ideally an orthopantomographic radiograph. The current study affirms the discoveries of the prior examinations.

\section{Compliance with ethical standards}

\section{Acknowledgments}

The author acknowledge Dr. Santosh Verma [Prof. and Head.] and Dr. Yukti Raj [Sr. lecturer] for their assistance and guidance. The author also like to thanks his colleagues Dr. Dhruv and Dr. Malvika for their valuable support.

\section{Disclosure of conflict of interest}

There is no conflict of interest.

\section{Statement of informed consent}

Informed consent was signed to all respondents before the data collection.

\section{References}

[1] Shah RM, Boyd MA and Vakil TF. (1978). Studies of permanent tooth anomalies in 7,886 Canadian individuals impacted teeth. J Can Dent Assoc, 44, 262-264.

[2] Grover PS and Lorton L. (1985). The incidence of unerupted permanent teeth and related clinical cases. Oral Surg Oral Med Oral Pathol, 59, 420-425.

[3] Javid B. (1985). Transmigration of impacted mandibular cuspids. IntJOral Surg, 14, 547-549.

[4] Ando S, Aizawa K, Nakashima T, Sanka Y, Shimbo K and Kiyokawa K. (1964). Transmigration process of the impacted mandibular cuspid. J Nihon Univ Sch Dent, 6, 66-71.

[5] Cakan DG, Ulkur F and Taner T. (2013). The genetic basis of dental anomalies and its relation to orthodontics. Eur J Dent, 7, 143-147.

[6] Peck S. (1998). On the phenomenon of intraosseous migration of nonerupting teeth. Am J Orthod Dentofacial Orthop, 113, 515-7.

[7] Mupparapu M. (2002). Patterns of intra-osseous transmigration and ectopic eruption of mandibular canines: review of literature and report of nine additional cases. Dentomaxillofac Radiol, 31, 355-360.

[8] Costello JP, Worth JC and Jones AG. (1996). Transmigration of permanent mandibular canines. Br Dent J, 181, 212-3.

[9] Joshi MR. (2001). Transmigrant mandibular canines: a record of 28 cases and a retrospective review of the literature. Angle Orthod, 71, 12-22.

[10] Kerr WJ. (1982). A migratory mandibular canine. Br J Orthod, 9, 111-112.

[11] Alaejos-Algarra C, Berini-Aytes L andGay-Escoda C. (1998). Transmigration of mandibular canines: report of six cases and review of the literature. Quintessence Int, 29, 395-398.

[12] Mitchell L. (1993). Displacement of a mandibular canine following fracture of the mandible. Br Dent J, 174, 417418.

[13] Nixon F and Lowey MN. (1990). Failed eruption of the permanent canine following open reduction of a mandibular fracture in a child. Br Dent J, 168, 204-205.

[14] Ranta R and Ylipaavalniemi P. (1973). The effect of jaw fractures in children on the development of permanent teeth and the occlusion. Proc Finn Dent Soc, 69, 99-104.

[15] Shapira Y, Mischler WA and Kuftinec MM. (1982).The displaced mandibular canine. ASDC J Dent Child, 49, 362364. 
[16] Shapira Y and Kuftinec MM. (1995). Intraosseous transmigration of mandibular canines-review of the literature and treatment options. Compend Contin Educ Dent, 16, 1014-1018.

[17] Taguchi Y, Kurol J, Kobayashi H and Noda T. (2001). Eruption disturbances of mandibular permanent canines in Japanese children. Int J Paediatr Dent,11, 98-102.

[18] Vichi M and Franchi L. (1991). The transmigration of the permanent lower canine. Minerva Stomatol, 40, 579589.

[19] Thoma KH. (1952). Oral Surgery. 2nd ed. St Louis, Miss: CV Mosby.

[20] Fiedler LD andAlling CC. (1968). Malpositioned mandibular right canine: report of case. J Oral Surg, 26, $405-407$.

[21] Wertz RA. (1994). Treatment of transmigrated mandibular canines. Am J Orthod Dentofacial Orthop, 106, 419427.

[22] Abbott DM, Svirsky JA and Yarborough BH. (1980). Transposition of the permanent mandibular canine. Oral Surg Oral Med Oral Pathol, 49, 97.

\section{How to cite this article}

Santosh K, Adeel AB, Yukti R, Dhruv KT and Malvika A. (2020). Transmigration of mandibular canines - A review of the literature and a report of five cases. World Journal of Advanced Research and Reviews, 6(3), 121-129. 